\title{
Influencia de la comunicación en una tarea cooperativa de representación espacial para maestras de educación infantil en formación
}

\author{
Ainhoa Berciano ${ }^{1}$ y Clara Jiménez-Gestal ${ }^{2}$ \\ (1) Departamento de Didáctica de la Matemática, Ciencias Experimentales y Sociales, Universidad del País \\ Vasco/Euskal Herriko Unibertsitatea, Leioa, España (correo-e: ainhoa.berciano@ehu.eus). \\ (2) Departamento de Matemáticas y Computación, Universidad de La Rioja, Logroño, España \\ (correo-e: clara.jimenez@unirioja.es).
}

Recibido Oct. 30, 2020; Aceptado Dic. 29, 2020; Versión final Feb. 14, 2021, Publicado Jun. 2021

\begin{abstract}
Resumen
Este artículo centra su interés en analizar la repercusión de la comunicación entre iguales en la resolución de tareas de representación espacial relacionadas con la orientación en contextos de aprendizaje cooperativo. Numerosas investigaciones destacan la necesidad de desarrollar correctamente el pensamiento espacial en las personas para que sean capaces de desenvolverse correctamente en su día a día. Dentro de esto, destaca la orientación, como la competencia relacionada con tareas de ubicación espacial. Desde un paradigma interpretativo, se realiza un estudio de casos con maestras de educación infantil en formación. Los resultados más destacados son que la comunicación entre iguales favorece y mejora la destreza de representación espacial siempre y cuando el tipo de comunicación tenga una componente reflexiva y comunicativa; no así, si la componente es puramente socio-afectiva. Se concluye que la comunicación entre iguales, siempre y cuando la comunicación sea de tipo reflexivo y comunicativo, mejora la resolución de tareas de representación relacionadas con la orientación.
\end{abstract}

\section{Influence of communication on a cooperative spatial representation task for prospective early childhood education teachers}

\begin{abstract}
This study focuses on analysing the impact of peer communication on the resolution of orientation-related spatial representation tasks in cooperative learning. Research studies have highlighted the need for people to develop spatial thinking correctly to properly perform day-to-day activities. Orientation is an important spatial thinking competence as it is related to spatial location tasks. From an interpretative paradigm, a case study is performed with female early childhood education teachers in training. The results show that communication between equals favours and improves spatial representation skills as long as the communication type has a reflexive and communicative component, but not if the component is purely affective. It is concluded that communication between peers improves the resolution of orientation-related representation tasks if the communication is reflexive and communicative.
\end{abstract}

Keywords: learning orientation; spatial representation; teachers training; childhood education; cooperative learning 


\section{INTRODUCCION}

En numerosas ocasiones, las entidades internacionales han destacado la necesidad de que los currículos escolares proporcionen los medios suficientes para que la educación sea capaz de dar respuesta a una sociedad en constante cambio. En este contexto, las Naciones Unidas aprobó en 2015 los Objetivos de Desarrollo Sostenible, entre los que se enmarca la educación de calidad, cuyo objetivo es que el alumnado desarrolle un pensamiento crítico y cívico, respetuoso con su entorno. Respecto al pensamiento crítico, algunas investigaciones plantean la relación existente entre éste y la competencia matemática y cómo la competencia matemática puede ayudar a su desarrollo (Su et al., 2016). Por su singular definición, en este trabajo centramos nuestro interés en el pensamiento o razonamiento espacial, debido al papel fundamental que juega en la adquisición de la competencia matemática (Young et al., 2018). Este pensamiento, entendido como una habilidad intrínsecamente humana, nos ayuda a razonar y entender el entorno físico en el que vivimos y constituye una parte fundamental de la competencia matemática. Según el National Research Council (2006), el pensamiento espacial es una competencia conformada por varias habilidades que a su vez capacitan para: entender conceptos espaciales, realizar tareas de representación, evaluar procesos y hacer razonamientos geométricos. Así, según Stiles y Stern (2001), el pensamiento espacial debe ayudarnos a poder responder a dos tipos de preguntas clave: qué objeto es y dónde se ubica. Este planteamiento lleva a poder describir el pensamiento espacial como la unión o amalgama de las capacidades visualización y orientación.

La visualización es la capacidad de "ver" o "imaginar" mentalmente los objetos geométricos espaciales, y la representación espacial asociada a la visualización se entiende como la capacidad de realizar tareas concretas con objetos, tales como rotaciones, representaciones planas, construcciones mentales de objetos desde sus desarrollos planos,...(Gonzato et al., 2011); mientras que la orientación, descrita como la habilidad de ubicarse en el espacio, involucra las siguientes tareas de representación: establecer relaciones posicionales entre objetos en el espacio, describir trayectorias o desplazamientos, realizar mapas, usar escalas, etc. (Gonzato et al., 2011). En investigaciones previas se ha demostrado la capacidad de visualización y de orientación esta relacionada con el rendimiento académico (Diezmann y Lowrie, 2009; Frick, 2019) y con el desarrollo de otras habilidades matemáticas (Young et al., 218); este hecho ha llevado a que se profundice en el análisis de estas dos competencias. Para el caso de la visualización, la mayoría de los primeros trabajos centraban su interés en estudiar la capacidad de estudiantes de distintas edades en la realización de tareas de representación espacial o de transformación de objetos mediante operaciones matemáticas, p.ej., simetrías, homotecias, traslaciones, ... (Stiles y Stern, 2001), pero los trabajos posteriores centran su interés en analizar la preferencia del alumnado por el uso de la capacidad de visualización en la resolución de tareas no geométricas, entre ellas, algebraicas o físicas (Arredondo et al., 2020; Ozkan et al., 2018) o por qué de su preferencia desde una perspectiva psicológica (Lowrie, et al. 2020).

Igualmente, en el caso de la orientación, son muchas las investigaciones realizadas desde distintas disciplinas, como queda patente en aquellas de otros ámbitos como puede ser la didáctica de la geografía (López y Martínez-Medina, 2015); pero al restringir nuestro interés al caso de la Didáctica de la Matemática, la mayoría de los trabajos analizan la capacidad de estudiantes en tareas de representación, destacando aquellas que se centran en plantear la realización de un mapa o plano. La selección de este tipo de tarea está claramente justificada por la gran cantidad de conocimientos matemáticos necesarios para su correcta realización: la determinación de un sistema de referencia pertinente, la necesidad de articular los diferentes planos obtenidos por la representación de los espacios locales situados alrededor de los puntos de referencia y la representación de los desplazamientos y su coordinación en el espacio considerado (Berthelot y Salin, 1992). Encontramos estudios que abordan el tipo de representación realizada en los planos y los conocimientos involucrados, destacando, entre otros, el análisis de los símbolos usados (Berciano et al., 2017; Torres y Climent, 2010). El nexo de todos estos estudios es que abordan la problemática desde la perspectiva del estudiante y no desde la perspectiva del profesorado y, mucho menos, desde la de la formación inicial del profesorado. Por tanto, con el fin de analizar este último aspecto, atendiendo a un enfoque socio constructivista de la educación, en el que el alumnado debe ser parte activa de su propio aprendizaje, centramos nuestro interés en el aprendizaje cooperativo.

El aprendizaje cooperativo se define como "el empleo didáctico de grupos reducidos en los que los alumnos trabajan juntos para maximizar su propio aprendizaje y el de los demás" (Johnson et al., 1999, p.7) y, para que se dé un aprendizaje cooperativo, el grupo debe tomar decisiones atendiendo a todos los puntos de vista, todas las personas del grupo deben ser consideradas iguales, se debe crear un clima de confianza y afectivo y debe haber comunicación intra-grupal, de modo que se gestionen los posibles conflictos que pudieran surgir. Además, en el aprendizaje cooperativo el objetivo está claramente definido por el docente (Panitz, 1999). Aunque tanto profesorado como alumnado tienen distintos puntos de vista sobre la repercusión del aprendizaje cooperativo en el aula (Opdecam y Everaert, 2018), son varias las 
investigaciones que ponen de manifiesto los efectos potenciales del mismo atendiendo a: el desarrollo del pensamiento creativo (Huff, 2014), la mejora de habilidades sociales (Mendo-Lázaro et al., 2018) y el aumento de la motivación intrínseca (Cecchini Estrada et al., 2019), entre otros. En este contexto, también se pueden encontrar investigaciones que analizan la repercusión del aprendizaje cooperativo en el aula de matemáticas desde: 1) la función docente (Ding et al., 2007), 2) el desarrollo actitudinal y de rendimiento del alumnado (Karali y Aydemir, 2018; Maure y Marimón, 2014; Chan e Idris, 2017); 3) el tipo de resolución de tareas matemáticas que realiza el alumnado; 4) el desarrollo de habilidades críticas-reflexivas (MoralesMaure et al., 2018); 5) la comparación con otras metodologías docentes (Pérez Estébanez, 2017).

Por otro lado, cuando restringimos nuestro interés a la comunicación en el aula de matemáticas, Morgan et al. (2014) mencionan la necesidad de investigar sobre comunicación oral del alumnado como medio para analizar el desarrollo de sus conocimientos matemáticos. Así, por ejemplo, en Murillo et al. (2011) señalan la complejidad que tiene el lenguaje geométrico en la comunicación oral, debido a que éste incluye tanto el uso de un lenguaje natural como la necesidad de comprender y describir correctamente conceptos geométricos que, a su vez, involucran diversos procesos cognitivos.

Pero hasta donde saben las autoras, son escasos los trabajos que analizan la repercusión del aprendizaje cooperativo en la realización de tareas de representación espacial asociadas a la orientación y, aún menos, aquellas que examinen la importancia de la comunicación entre iguales en la resolución de tareas matemáticas en contextos de aprendizaje. Por tanto, el objetivo de nuestra investigación es analizar cómo influye el aprendizaje cooperativo en la resolución grupal de una tarea de representación espacial, frente a la realización individual de la misma tarea. En concreto, nuestro interés se centra en analizar qué tipo de comunicación surge de modo natural entre iguales y qué tipo de variaciones produce este tipo de comunicación en la realización de la tarea matemática. Así, desde un paradigma interpretativo, se ha realizado un estudio cualitativo descriptivo del tipo de representación individual y grupal realizada por maestras y maestros de Educación Infantil en formación y de la repercusión que tiene la comunicación entre iguales en la realización de la tarea grupal en un contexto de aprendizaje colaborativo. De este análisis, el resultado más destacado que se desprende es la estrecha relación entre el tipo de comunicación entre iguales que se produce en el aprendizaje cooperativo y el tipo de mejora en la realización de la tarea encomendada.

\section{METODOLOGÍA}

Presentamos la metodología en dos subsecciones: en la primera, se presenta el diseño del experimento de enseñanza, los participantes y el desarrollo del mismo; en la segunda, se describen los instrumentos construidos para realizar la recogida de datos y posterior análisis de la información.

\section{Diseño del experimento de enseñanza}

El experimento de enseñanza (Bernabeu et al., 2019) está diseñado para maestras y maestros de Educación Infantil en formación inicial, dentro de la asignatura Pensamiento matemático y su didáctica, que es la única asignatura obligatoria relacionada con la enseñanza-aprendizaje de las matemáticas de la titulación universitaria. Entre los resultados de aprendizaje de la asignatura, presentes también en otros grados en Educación Infantil españoles, destaca la capacidad de conocer herramientas y recursos didácticos para desarrollar las habilidades matemáticas, en concreto las espaciales y geométricas, así como desarrollar las habilidades comunicativas. Con esto en mente, se diseñó el experimento de enseñanza que presentamos para alcanzar el objetivo de nuestra investigación; esto es, analizar qué tipo de comunicación surge de modo natural entre iguales y qué tipo de variaciones produce este tipo de comunicación en la realización de la tarea matemática.

Participaron un total de 20 estudiantes del grado de Educación Infantil, todas mujeres excepto un hombre. Se formaron 4 grupos de tres personas y dos de cuatro. Se analizaron las producciones de los grupos y, para este trabajo, se presentan los resultados de los dos grupos de cuatro personas, uno con resultados positivos y otro con resultados negativos. Para la tarea de representación espacial, se propuso la elaboración de un plano de un entorno conocido. Se escogió el aula-taller de matemáticas, distribuyendo las mesas de manera que sus proyecciones verticales compusieran al menos tres formas geométricas diferentes (rectángulo, hexágono y corona hexagonal) y se introdujeron diversos objetos distractores (juegos y materiales en la repisa de la ventana, instrumentos de dibujo en la de la pizarra y botellas de diferentes formas y capacidades encima del armario). La disposición de los objetos de la clase, distinta de la habitual, y la presencia de los distractores pretenden valorar si las participantes son capaces de reconocer los contenidos geométricos necesarios para la elaboración del plano obviando lo superfluo.

Los instrumentos de dibujo proporcionados fueron, hojas DIN-A3, reglas, escuadras, cartabones, lapicero, goma de borrar, sacapuntas y pinturas de colores. Para realizar las medidas se disponía de cintas métricas 
y estaba permitido moverse por todo el recinto. En una sesión de dos horas, se dieron a las estudiantes las siguientes instrucciones: 1) En la hoja DIN-A3 que se os ha proporcionado debéis dibujar un plano del aula, en la que localicéis todos los elementos que sean significativos para identificarla; 2) tomad todos los datos que consideréis necesarios, podéis moveros por la clase y utilizar los instrumentos de medida; 3 ) posteriormente, en grupos, discutiréis sobre los planos realizados individualmente, y cada una explicará al resto de compañeras el procedimiento seguido para dibujar su plano, para lo que dispondrá de la fotocopia de su plano; 4) finalmente, dibujaréis, entre todas, un nuevo plano grupal en DIN-A3.

\section{Instrumentos para la recogida y análisis de información}

La recogida de datos se realizó utilizando los planos elaborados, tanto individuales como colectivos, y las grabaciones en audio del trabajo grupal. También se revisaron las anotaciones efectuadas, durante la realización de la actividad, en el cuaderno de notas de las investigadoras. Los datos se analizaron mediante dos instrumentos diseñados ad hoc, atendiendo específicamente a los aspectos relevantes de la actividad, con la posterior triangulación de los datos (esta triangulación consistió en comprobar el grado de concordancia en la clasificación de la corrección de los planos y en la clasificación de los tipos de conversación entre iguales por personas expertas del área). Para el análisis de los planos, siguiendo las orientaciones presentadas en el trabajo de Berthelot y Salin (1992), se tuvieron en cuenta indicadores de: proporción, tanto del aula como del mobiliario; forma; perspectiva, tipo de proyección utilizada; orientación; y pertinencia y relevancia de los objetos representados. Así mismo, para cada indicador se especificaron descriptores que se pueden considerar asociados a distinto nivel de comprensión de la representación de planos. La tabla 1 recoge los indicadores y sus descriptores asociados.

Tabla 1: Indicadores y descriptores usados en el análisis de los planos individuales y grupales

\begin{tabular}{|l|l|}
\hline \multicolumn{1}{|c|}{ Indicadores } & \multicolumn{1}{c|}{ Descriptores } \\
\hline Proporción & $\begin{array}{l}\text { Estima las proporciones de los principales elementos arquitectónicos del aula } \\
\text { Utiliza una escala para representar las medidas }\end{array}$ \\
\hline Proporción mobiliario & $\begin{array}{l}\text { Mantiene proporciones con las dimensiones del aula } \\
\text { Los objetos mantienen las proporciones entre sí }\end{array}$ \\
\hline Formas & Se identifican las formas de los objetos que representan \\
\hline Perspectiva & $\begin{array}{l}\text { Representa los objetos mediante una única proyección } \\
\text { Mezcla dos proyecciones }\end{array}$ \\
\hline Orientación & $\begin{array}{l}\text { Identifica los diferentes elementos } \\
\text { Respeta la ubicación de los objetos }\end{array}$ \\
\hline Pertinencia & $\begin{array}{l}\text { Identifica todos los elementos relevantes para realizar el plano } \\
\text { Faltan elementos relevantes (puertas, ventanas, ...) } \\
\text { Identifica elementos irrelevantes }\end{array}$ \\
\hline
\end{tabular}

Respecto a las interacciones grupales y el análisis de la conversación, se utilizó como marco de referencia las funciones de la comunicación oral definidas por Jakobson (1960). Atendiendo a la clasificación original (1. Función referencial, descripción de una situación; 2. función metalingüística, sobre el uso del lenguaje; 3. función emotiva, enfatiza sentimientos de la persona emisora; 4. función poética: se centra en el formato estético de la comunicación; 5 . función conativa, busca una respuesta en la persona receptora; 6 . función fática, busca establecer un canal de interlocución) y a las características de la comunicación (entre iguales), se ha prestado una mayor atención en aquellas que emergen de modo natural en estos contextos. Así, se consideraron los aspectos asociados a la función referencial (desglosada a su vez en: 1. componente comunicativa, cuando la intención es exponer o describir su propio plano; 2. componente reflexiva, cuando se trata de persuadir o proponer cambios) y los asociados a las funciones emotivas y conativas agrupadas en la llamada componente socio afectiva, con el fin de mejorar el ambiente del grupo. Para cada componente, en la tabla 2 se reflejan los descriptores considerados.

Tabla 2: Componentes y descriptores usados en el análisis de las conversaciones grupales

\begin{tabular}{|l|l|}
\hline Componente & \multicolumn{1}{c|}{ Descriptores } \\
\hline Comunicativa & $\begin{array}{l}\text { Utiliza vocabulario geométrico } \\
\text { El vocabulario se corresponde con las acciones matemáticas que describe }\end{array}$ \\
\hline Reflexiva & $\begin{array}{l}\text { Propone variaciones en el plano común tras escuchar las intervenciones de las compañeras } \\
\text { Se plantea el sentido de la actividad y lo comunica }\end{array}$ \\
\hline Socio afectiva & $\begin{array}{l}\text { Escucha las intervenciones de las compañeras y las tiene en cuenta } \\
\text { Alienta y respalda a sus compañeras en la realización de tareas matemáticas }\end{array}$ \\
\hline
\end{tabular}




\section{RESULTADOS}

Cinco de los grupos mejoraron la producción grupal tras la discusión sobre los planos individuales y solo uno empeoró. La tabla 3 recoge los resultados de los cuatro grupos que completan la investigación aquí presentada, pero con el fin de dar respuesta al objetivo de investigación, y analizar en profundidad el porqué de la variación en las producciones, sólo presentamos en detalle los resultados de los dos grupos formados por cuatro personas, en uno de ellos se mejoró la producción final y en el otro empeoró. Las estudiantes están etiquetadas con los nombres NX, donde $\mathrm{N}$ indica el número del grupo y $\mathrm{X}$ es una letra de la $\mathrm{A}$ a la $\mathrm{D}$.

Tabla 3: Resumen de los resultados del resto de los grupos

\begin{tabular}{|c|c|c|c|c|c|c|}
\hline \multirow{2}{*}{ Código } & \multicolumn{6}{|c|}{ Tipos de errores } \\
\hline & Proporción & Mobiliario & perspectiva & Formas & Orientación & Pertinencia \\
\hline $3 \mathrm{~A}$ & & $x$ & & & & \\
\hline $3 \mathrm{~B}$ & $x$ & $x$ & & & & $x$ \\
\hline $3 C$ & & & & & & $x$ \\
\hline $3 G$ (grupo) & & $x$ & $x$ & & & $x$ \\
\hline $4 \mathrm{~A}$ & & $x$ & $x$ & & & $x$ \\
\hline $4 \mathrm{~B}$ & $x$ & $x$ & $x$ & $x$ & & \\
\hline $4 \mathrm{C}$ & $x$ & $x$ & $x$ & & & $x$ \\
\hline 4G (grupo) & & & & & & $x$ \\
\hline $5 \mathrm{~A}$ & $x$ & $x$ & $x$ & $x$ & & $x$ \\
\hline $5 \mathrm{~B}$ & $x$ & $x$ & & & & $x$ \\
\hline $5 \mathrm{C}$ & $x$ & $x$ & $x$ & & & \\
\hline $5 G$ (grupo) & & $x$ & $x$ & & & \\
\hline $6 \mathrm{~A}$ & $x$ & $x$ & $x$ & & & $x$ \\
\hline $6 \mathrm{~B}$ & $x$ & $x$ & & & & $x$ \\
\hline $6 \mathrm{C}$ & $x$ & $x$ & $x$ & & & $x$ \\
\hline 6G (grupo) & $x$ & & & & & \\
\hline
\end{tabular}

\section{Resultados para el grupo 1}

En los planos individuales del grupo 1 se observó que presentan diferentes características. La estudiante $1 \mathrm{~A}$ presenta un error grave de orientación, su plano individual es el único en el que se han invertido las dimensiones de la clase, y manifiesta errores en todos los indicadores, exceptuando que no mezcla perspectivas. Otro miembro, 1B, identifica los elementos relevantes y los orienta adecuadamente, sin embargo, no mantiene las proporciones ni del aula ni de los objetos y confunde formas. Por su parte, 1C no mantiene las proporciones ni reproduce la forma de la mesa central, que deja inacabada, aunque plasma con detalle algunos de los distractores. Por último, 1D evidencia el concepto de escala y mantiene las proporciones del recinto, aunque no las de los objetos que previamente no ha dimensionado. Su plano no está completo. Las transcripciones de las grabaciones nos revelan que dos de los miembros reconocen haber usado las baldosas como unidad de medida, aunque posteriormente una de ellas no sabe utilizar las medidas para plantear una escala y realizar el dibujo: 1D: He medido por pasos y baldosa. Las baldosas miden entre 40 y $42 \mathrm{~cm}$ yo les he dado 40, luego donde estaban las cosas y con una escala aproximada cada $40 \mathrm{~cm}$ de la realidad he puesto una baldosa. Voy poniendo las partes fijas, ... 1B: He medido por baldosa y luego he contado y he puesto "más o menos a ojo".

En la explicación de su plano, $1 \mathrm{~A}$ atribuye el error de orientación del plano a que, una vez comenzado el dibujo de la pared larga por el lado estrecho del folio, ya no le cabía toda el aula y decide invertirla. Aun antes de que sus compañeras se lo adviertan, ella observa su error y se da cuenta de la importancia de mantener las proporciones. La tabla 4 recoge los errores detectados al analizar los planos realizados y las descripciones, relacionados con cada uno de los indicadores.

Tabla 4: Errores según la dimensión del grupo 1

\begin{tabular}{|l|l|l|l|l|l|l|}
\hline \multirow{2}{*}{ Código } & \multicolumn{7}{|l|}{ Tipos de errores } & \\
\cline { 2 - 7 } & Proporción & Mobiliario & Perspectiva & Formas & Orientación & Pertinencia \\
\hline 1A & $\mathrm{x}$ & $\mathrm{x}$ & $\mathrm{x}$ & & $\mathrm{x}$ & $\mathrm{x}$ \\
\hline 1B & $\mathrm{x}$ & $\mathrm{x}$ & $\mathrm{x}$ & & & \\
\hline 1C & & $\mathrm{x}$ & & & $\mathrm{x}$ & $\mathrm{x}$ \\
\hline 1D & & $\mathrm{x}$ & & & & $\mathrm{x}$ \\
\hline
\end{tabular}


En el plano grupal se advierte una gran mejoría, aparecen más elementos que en ninguno de los planos individuales (las sillas apiladas en las esquinas, las puertas de los almacenes, la papelera-paragüero). Mejoran las proporciones del aula y no se reproduce ninguno de los errores que se aprecian en los cuatro planos individuales. En el proceso de elaboración del plano conjunto se ponen de acuerdo rápidamente en la elección de la persona que va a dibujar, atendiendo a lo que consideran que es el plano individual mejor dibujado, y enseguida comienza el proceso. En la discusión, comienzan acordando la escala del mapa, tomando como medida las baldosas y representándolas por un centímetro. Con esta medida, dibujan el contorno de la clase, paredes, puertas, ventanas y los objetos anexos. Para ubicar los objetos dentro del aula acuerdan utilizar un sistema de referencia en el que el origen sea la mesa situada en el centro de la clase y colocar el resto de objetos, comenzando por los más grandes, para terminar con los más pequeños: 1D: Igual las mesas, lo tenemos que hacer; 1B: Sí, igual primero haría lo grande y luego los pequeños detalles yo lo haría, igual, al final; 1D: A ver, la sala para hacer referencia. La mesa del centro está prácticamente en el centro del aula ¿habéis medido?; 1C: Sí, yo creo que sí.

\section{Resultados para el grupo 2}

Los planos elaborados por los componentes del segundo grupo también muestran diferencias. La estudiante $2 \mathrm{~A}$ ha utilizado las baldosas como unidad de medida de las longitudes de la clase, pero no ha respetado las proporciones ni en las dimensiones de la sala ni con el mobiliario. Tampoco 2B mantiene proporciones ni entre las mesas iguales ni entre objetos próximos como las puertas, aunque representa todos los objetos pertinentes. La estudiante $2 \mathrm{C}$ mantiene buenos indicadores en lo que respecta a proporciones y localización de los objetos, pero obvia elementos significativos e introduce el color. En el plano de 2D, destaca la mezcla de perspectivas para poder dibujar los detalles.

En la descripción de los planos, tres de las componentes de este grupo también manifiestan haber tomado como referencia las baldosas mientras que $2 \mathrm{~B}$ reconoce que no las ha contado y que esto ha influido en el resultado. 2B- Vale, pues yo, a diferencia de mis compañeras no he contado las baldosas y a la hora de hacer el plano lo he hecho más a ojo y me he dado cuenta de que no queda tan bien. He dibujado la puerta de la entrada, también la puerta que da al almacén [...] Luego también vendría el segundo almacén, la puerta la he representado simplemente con un espacio porque es corredera y no sabía cómo se representaba. [...] He dibujado las mesas más o menos desde mi posición según iba viéndolas, he dibujado también al lado del ventanal un montón de sillas que había, nada, con un rectángulo pequeñito, he usado también un rectángulo pequeño para la pizarra, y luego para acabar con un rectángulo también he representado el proyector con la mesa del ordenador.

En su descripción muestra sus dudas a la hora de representar determinados elementos del aula y especifica las figuras geométricas que utiliza para representar los distintos objetos. En la explicación del plano de 2C aparece un componente de reflexivo sobre la intención de la actividad que nada tiene que ver con la descripción geométrica: $2 \mathrm{C}$ : He contado las baldosas y he intentado ajustarme al dibujar el plano. Luego he seguido realizando el armario [...] intentando ajustarme lo más posible [...] y luego, mientras lo estaba haciendo, estaba pensando en algo, en la mesa, aparte de una regla, de una goma y un lápiz, también nos han dejado pinturillas y nos han dicho que nos ajustemos lo más posible a la realidad. Pero, no sé, mientras estaba realizando el dibujo, también estaba pensando, si nos han dejado las pinturillas y esto se lo pidiéramos a nuestros niños, igual no lo harían exacto [...] igual el objetivo no es medir si es una baldosa o dos, sino igual la perspectiva o cómo ves tú el dibujo [...] Los niños lo van a ver desde su perspectiva y eso no va a ser con reglas, ni midiendo ni nada, sino simplemente va a ser para hacer un dibujo con pinturillas como ellos se expresan. Me ha hecho recapacitar y en vez de pintar las mesas me he puesto a colorear [...] Que no sé si ese es el objetivo. [...] igual el objetivo no es tanto de contar, sino cómo lo vemos nosotros. Esa es mi teoría. La tabla 5 recoge los errores detectados al analizar los planos realizados, relacionados con cada uno de los indicadores.

Tabla 5: Errores según la dimensión del grupo 2

\begin{tabular}{|l|l|l|l|l|l|l|}
\hline \multirow{2}{*}{ Código } & \multicolumn{5}{l|}{ Tipos de errores } \\
\cline { 2 - 7 } & Proporción & Mobiliario & perspectiva & Formas & Orientación & Pertinencia \\
\hline 2A & $\mathrm{x}$ & $\mathrm{x}$ & & & & $\mathrm{x}$ \\
\hline 2B & $\mathrm{x}$ & $\mathrm{x}$ & $\mathrm{x}$ & $\mathrm{x}$ & & \\
\hline 2C & $\mathrm{x}$ & $\mathrm{x}$ & & & $\mathrm{x}$ & $\mathrm{x}$ \\
\hline 2D & $\mathrm{x}$ & $\mathrm{x}$ & $\mathrm{x}$ & $\mathrm{x}$ & $\mathrm{x}$ & $\mathrm{x}$ \\
\hline 2G (grupo) & $\mathrm{x}$ & $\mathrm{x}$ & & $\mathrm{x}$ & & $\mathrm{x}$ \\
\hline
\end{tabular}


En este caso observamos que el plano realizado entre todas es sensiblemente inferior a los individuales, de hecho, ni lo terminan por falta de tiempo, pero dibujan y colorean elementos que no son necesarios para la identificación del aula. Para la elaboración del plano conjunto, en este grupo se pone de manifiesto la inseguridad de sus componentes sobre sus propias representaciones y antes de tomar la decisión de quién va a dibujar recuerdan el procedimiento seguido por cada una. 2B: Entonces, ahora que hemos visto cada una nuestros planos tenemos que hacer uno. Y habrá que elegir quién lo hace. Yo dibujo fatal; 2D: Sí pues, mira mis mesas; 2B: A mi me ha resultado "superdifícil" hacer las mesas; 2A: Y a mi también; 2C: Bueno, si no, puede empezar una y luego si vemos que resulta difícil pues 2D-vale, pero tenemos, también que decidir a ver cuál es el que mejor está hecho. Como representar, o cada parte, igual de cada dibujo encontramos; 2B; Vosotras para hacer el vuestro ¿por dónde habéis empezado?; 2D: Yo por aquí yo por el proyector y por la puerta; $2 \mathrm{C}$ : Yo he empezado bueno, primero hecho lo que es el contorno del aula, he hecho primero un rectángulo y luego he ido quitando las medidas; 2D: Bueno, yo también he empezado con el contorno, pero luego después con el proyector. Bueno, aunque no se vea bien aquí el contorno aquí había [...]; 2A: Pues vamos a hacer rápido lo que es la sala y así y luego nos centramos en los detalles.

En el proceso de elaboración del plano conjunto tienen en cuenta las elaboraciones previas. 2A: ¿Qué medidas has tomado más o menos? para hacer; 2C: No sé, pero por ejemplo la de 2B también está bien y tiene justo las medidas puestas, se puede seguir esas medidas. $Y$ todas participan en la toma de decisiones sobre las medidas: 2A: Dos y medio, vale y ahora dieciséis para arriba; 2D: Dieciséis, vale ¿y de aquí a aquí cuántos eran?; 2B: Yo creo que dieciocho, pero...; 2C: Yo he puesto catorce; 2D: Puede ser que haya dieciocho. Porque catorce he pasado ya. [...]; 2C: Bueno pues pon 16 y así, y ahora borrar lo que falta o ponte una marquita aquí y borras; 2A: Pero de fondo, no has contado 16 así, ¿en el fondo de la ventana? Ah, desde ahí, vale. Vale, sí, que claro; 2C: Vale y ahora ya haz la recta si quieres; 2A: Ah, no, no porque tenemos que hacer la entrada; 2B: hay que hacer el cubito; 2C: eso es ¿cuanto cuando tiene eso?; 2A: yo había dicho seis que es lo mismo que esto. [...] Porque aquí íbamos a poner nueve y medio; 2C: Entonces no corresponde esto, porque si aquí hemos ampliado y aquí hemos dicho que es exacto. ¿No?; 2a․ No, no hemos ampliado; 2C: Sí, pero, por ejemplo, de aquí habéis dicho que eran 18 y habéis puesto 18. ¿Y porque aquí 10? ¿Por qué son 10 baldosas?; 2A: No, no son diez. Aquí no hemos seguido el número real. Serían cinco y seis.

Ante las dudas de la persona que está dibujando sus compañeras la animan: 2D: Es que, claro, así contrarreloj no me da tiempo, el cable va así para atrás y aquí había hecho como tiene. Cómo tiene la base redonda así, pero que no se si está bien así o qué; $2 \mathrm{C}$ : Pero que yo creo que está genial, aunque la base [...]; 2D: Porque claro, no da tiempo; 2C: Bueno, tranquila no pasa nada, a ver que es una actividad; 2B: Eso es; 2D: Ya, pero...; 2A: Ya, ya, ya sé como eres; 2B: Bueno, tranquila mujer; 2C: Si la cuestión es ponernos de acuerdo y realizar el plano como creemos; 2D: ¿y cómo y cómo hacemos? En un momento del proceso reflexionan sobre lo que habrían dibujado los niños en la actividad, lo que resulta curioso porque en el enunciado de la actividad no se hace referencia a ello: 2B: Siguiendo tu teoría ¿sabes qué también dibujarían los niños?; 2C: ¿qué?; 2B: A los compañeros; 2C: Claro y luego igual no lo dibujarían desde arriba, dibujarían lo que ven ellos desde la perspectiva en la que están sentados; 2B: Pero eso no sería un plano.

En todo el desarrollo de la actividad se han producido interacciones entre las componentes del grupo respecto a los aspectos formales, la necesidad de dibujar las puertas u otros elementos de la clase, cómo representarlos, por dónde empezar, la cantidad de detalles y colores necesarios en el dibujo; así como respecto a cuestiones afectivas, animando a la persona que dibuja, apoyándola en sus decisiones, tranquilizándola y recalcando los aspectos positivos de su trabajo.

\section{DISCUSIÓN}

Un análisis pormenorizado de los resultados anteriormente expuestos nos permite afirmar que todas las alumnas utilizaron sus conocimientos geométricos para abordar la tarea planteada. En general, en todos los planos elaborados se mantienen las propiedades topológicas y afines. Las mayores dificultades se manifiestan en la aplicación de las propiedades métricas, y más concretamente, en el uso de las proporciones tanto del aula como del mobiliario. La comunicación entre iguales ha tenido efectos diversos dependiendo del grupo; así, vemos cómo en uno las mejoras son claras, pero en otro no. Como no ha habido intervención alguna, de acuerdo con Murillo et al. (2011), podemos atribuir a la comunicación entre iguales este efecto. Por otro lado, y del mismo modo que en otras investigaciones, el abordaje tanto de las tareas personales como de las grupales y la comunicación de los procesos se fundamenta más en criterios socio-afectivos que en técnico-matemáticos (Chan e Idris, 2017; Karali y Aydemir, 2018). Una buena parte de las comunicaciones en los grupos se centran en aspectos socio afectivos, en los que se dedican a animarse mutuamente y a fortalecer su autoestima. 
En el grupo que muestra un plano grupal matemáticamente menos exacto, la discusión sobre aspectos irrelevantes para el objetivo arrastra a las componentes del mismo a la perplejidad y a un resultado significativamente peor que el conseguido por cada uno de sus miembros. Aunque individualmente han considerado el elemento distractor irrelevante, al plantearlo una de las compañeras, afloran sus sentimientos y se dejan arrastrar por las relaciones sociales obviando el objetivo. Igualmente se aprecia también que la toma de decisiones acerca de cómo hay que realizar la representación se ve retrasada por las diferentes observaciones de cada una de las integrantes y su confrontación, esto hace que no les de tiempo de realizar la representación completa. Sin embargo, el grupo que ha realizado un plano grupal más correcto que los individuales ha tenido una comunicación con componentes reflexiva y comunicativa mucho más efectiva, las afirmaciones realizadas por cada integrante se ajustan a las necesidades de la tarea y las observaciones e indicaciones que se hacen a la persona que dibuja el plano conjunto son más precisas, lo que les ha permitido realizar una tarea más correcta a nivel matemático.

Así, a pesar de que la tarea planteada basada en aprendizaje cooperativo ha mostrado un resultado desigual dependiendo del tipo de comunicación entre iguales; consideramos que ha mostrado un potencial significativo en la mejora de la resolución de tareas de representación relacionadas con la orientación, siempre y cuando la comunicación haya sido de tipo reflexivo y comunicativo. De hecho, la componente de reflexión del alumnado ha implicado el uso de destrezas meta cognitivas y en ella han aparecido tanto habilidades creativas como actitudes críticas hacia los propios medios de resolución individual de la actividad (Morales-Maure et al., 2017; Chan e Idris, 2017; Karali y Aydemir, 2018 ).

En la comunicación grupal también aparece un aspecto crítico sobre la posible transferencia de la actividad al aula de Educación Infantil, por lo que entendemos que este tipo de experiencias de enseñanzaaprendizaje funcional, incardinadas en su entorno cotidiano, en el que tienen que observar y aplicar los datos que ellas mismas recogen, les permitirán modificar su concepción de la enseñanza-aprendizaje de las matemáticas en su futura praxis (Ding et al., 2017; Maure y Marimón, 2014). Necesitamos encontrar un equilibrio entre los métodos que fomentan el aprendizaje del conocimiento matemático y los que potencian el desarrollo de habilidades socio-afecticas en la formación de maestras. Participar de enfoques pedagógicos que promuevan el aprendizaje activo y el pensamiento crítico capacitará a las estudiantes para proponer en su actividad profesional situaciones de aprendizaje adaptadas al medio infantil, contextualizadas en la vida real, pero a su vez, la investigación aquí planteada nos lleva a reflexionar sobre la necesidad de intervención profesional en el aula y la importancia de considerar en qué medida la comunicación entre iguales debe estar modelada por la figura del docente del aula.

Entre las características de la actividad que hemos analizado, destacamos su aplicabilidad en todas las áreas del currículo escolar básico y la necesidad de obtener datos para aplicarlos. También queremos resaltar que, con diferentes enfoques, puede plantearse desde los primeros años en la Educación Infantil, en la enseñanza básica (ver currículo), en la Educación Secundaria (Torres y Climent, 2010) y en la Universidad. Finalmente, no debemos obviar las limitaciones que posee el estudio. Entre ellas, destacamos que algunos aspectos relacionados con las variables de análisis no se han podido incorporar a este artículo, mostrando un análisis parcial y que plantea la necesidad de una mayor profundidad (por ejemplo, ¿Cómo varían la proporción, el uso de la simbología, la perspectiva, etc.?). Igualmente, el tamaño de la muestra usada es pequeño, lo que lleva a plantear si la mejora pudiera haberse debido a otro u otros factores latentes (por ejemplo, el tiempo). Todas estas limitaciones nos llevan a plantear futuras líneas de investigación que den respuestas a esta y otras preguntas de interés; para ello, se plantea la necesidad de realizar una investigación con una muestra mucho más amplia, donde se tenga un grupo de control y uno de investigación en el que se puedan comparar los resultados y analizar a un nivel más pormenorizado sus diferencias y los porqués de las mismas.

\section{CONCLUSIONES}

De acuerdo al trabajo presentado y a los resultados obtenidos, se pueden plantear las siguientes conclusiones principales:

1.- La comunicación entre iguales, siempre y cuando la comunicación sea de tipo reflexivo y comunicativo, mejora la resolución de tareas de representación relacionadas con la orientación.

2.- Las experiencias de enseñanza-aprendizaje funcional contextualizadas favorecen la reflexión sobre la práctica docente y su concepción de la enseñanza-aprendizaje.

3.- Surge la necesidad de encontrar un equilibrio entre los métodos que fomentan el aprendizaje del conocimiento matemático y los que potencian el desarrollo de habilidades socio-afecticas en la formación de maestras. 


\section{AGRADECIMIENTOS}

Esta investigación ha sido parcialmente financiada por las Ayudas a la investigación de la Universidad del País Vasco (UPV/EHU): Mod. II Grupos (códigos GIU19/008 y PPGA20/14).

\section{REFERENCIAS}

Arredondo, E.H., García-García, J.I., y Márquez Torres, M., La modelación metafórica del movimiento por estudiantes universitarios, http://dx.doi.org/10.4067/S0718-50062020000300055, Formación Universitaria, 13(4), 55-64 (2020)

Berciano, A., Jiménez-Gestal, C., y Salgado Somoza, M., Kindergartners' use of symbols in the semiotic representation of 3-dimensional changes, International Electronic Journal of Mathematics Education, 12(3), 311-331 (2017)

Bernabeu, M., Moreno, M., y Llinares, S., Experimento de enseñanza como una aproximación metodológica a la investigación en educación matemática, https://doi.org/10.17533/udea.unipluri.19.2.07, Uni-pluriversidad, 19(2), 103-123 (2019)

Berthelot, R., y Salin, M.H., L'enseignement de l'espace et de la géométrie dans la scolarité obligatoire, These, Université de Bourdeaux, Francia (1992)

Cecchini Estrada, J.A., González González-Mesa, C., y otros 3 autores, The impact of cooperative learning on peer relationships, intrinsic motivation and future intentions to do sport, https://doi.org/10.7334/psicothema2018.305, Psicothema, 31(2), 163-169 (2019)

Chan, L.L., y Idris, N., Cooperative learning in mathematics education, International Journal of Academic Research in Business and Social Sciences, 7(3), 539-553 (2017)

Diezmann, C. M., y Lowrie, T., Primary students' spatial visualization and spatial orientation: an evidence base for instruction, in Proceedings of the 33rd Conference of the International Group for the Psychology of Mathematics Education, 19-24, Aristotle University of Thessaloniki, Greece (2009)

Ding, M., Li, X., Piccolo, D., y Kulm, G., Teacher interventions in cooperative-learning mathematics classes, http://dx.doi.org/10.3200/JOER.100.3.162-175, The Journal of Education Research, 100(3), 162-175 (2007)

Frick, A., Spatial transformation abilities and their relation to later mathematics performance, https://doi.org/10.1007/s00426-018-1008-5, Psychological Research, 83(7), 1465-1484 (2019)

Gonzato M., Godino, J.D., y Neto, T., Evaluación de conocimientos didáctico-matemáticos sobre la visualización de objetos tridimensionales, Educación Matemática, 23, 5-37 (2011)

Huff, P., The goal project: a group assignment to encourage creative thinking, leadership abilities and communication skills, https://doi.org/10.1080/09639284.2014.974198, Accounting Education, 23(6), 582-594 (2014)

Jakobson, R., Closing statement: lingüistics and poetics, in Style in Language by T.A. Sebeok, 350-377, Jonsons Wiley \& Sons, Inc., New York, Estados Unidos (1960)

Johnson, D.W., Johnson, R.T., y Holubec, E.J., El aprendizaje cooperativo en el aula, Editorial Paidós, Buenos Aires, Argentina (1999)

Karali, Y., y Aydemir, H., The effect of cooperative learning on the academic achievement and attitude of students in mathematics class, https://doi.org/10.5897/ERR2018.3636, Educational Research and Reviews, 13(21), 712-722 (2018)

López Fernández, J.A., y Martínez Medina, R., La representación espacial en la formación inicial del profesorado de educación primaria, in Análisis espacial y representación geográfica: innovación y aplicación by J. De la Riva, P. Ibarra, R. Montorio y M. Rodrigues, 1435-1443, Universidad de Zaragoza-AGE, España (2015)

Lowrie, T., Resnick, I., Harris, D., y Logan, T., In search of the mechanisms that enable transfer from spatial reasoning to mathematics understanding, https://doi.org/10.1007/s13394-020-00336-9, Mathematics Education Research Journal, 32, 175-188 (2020)

Maure, L.M., y Marimón, O.G., Examining the role of college student's approach to math, https://doi.org/10.5897/ERR2014.1923, Educational Research and Reviews, 9(19), 761-770 (2014)

Mendo-Lázaro, S., León-del-Barco, B., y otros 3 autores, Cooperative team learning and the development of social skills in higher education: the variables involved, https://doi.org/10.3389/fpsyg.2018.01536, Frontiers in Psychology, 22(9), 1536, 1-11 (2018)

Morales-Maure, L., García-Marimón, O., Torres-Rodríguez, A., y Lebrija-Trejos, A., Habilidades cognitivas a través de la estrategia de aprendizaje cooperativo y perfeccionamiento epistemológico en matemática de estudiantes de primer año de universidad, http://dx.doi.org/10.4067/S0718-50062018000200045, Formación Universitaria, 11(2), 45-56 (2018)

Morgan, C., Craig, T., Schuette, M., y Wagner, D., Language and communication in mathematics education: an overview of research in the field, https://doi.org/10.1007/s11858-014-0624-9, The International Journal of Mathematics Education, 46(6), 843-853 (2014)

Murillo, J., Marcos, G., y Fortuny J.M., Una metodología de análisis de competencias en un entorno interactivo, Campo abierto. Revista de educación, 30(1), 11-34 (2011) 
National Research Council, Learning to think spatially, https://doi.org/10.17226/11019, The National Academies Press Washington, DC, Estados Unidos (2006)

Opdecam E., y Everaert, P., Seven disagreements about cooperative learning, https://doi.org/10.1080/09639284.2018.1477056, Accounting Education, 27(3), 223-233 (2018)

Ozkan, A., Esra Arikan, E., y Mehmet Ozkan, E., A study on the visualization skills of 6th grade students, https://doi.org/10.13189/ujer.2018.060219, Universal Journal of Educational Research, 6(2), 354-359 (2018)

Panitz, T., Collaborative versus cooperative learning: a comparison of the two concepts which will help us understand the underlying nature of interactive learning, https://files.eric.ed.gov/fulltext/ED448443.pdf, (1999)

Parzysz, B., Representation of space and students' conceptions at high school level, https://doi.org/10.1007/BF00312716, Educational Studies in Mathematics, 22, 575-593 (1991)

Stiles, J., y Stern, C., Developmental change in spatial cognitive processing: complexity effects and block construction performance in preschool children, http://dx.doi.org/10.1207/S15327647JCD0202_3, Journal of Cognition and Development, 2(2), 157-187 (2001)

Su, H.F., Ricci, F.A., y Mnatsakanian, M., Mathematical teaching strategies: pathways to critical thinking and metacognition, Journal of Research in Education and Science (IJRES), 2(1), 190-200 (2016)

Torres, J.M., y Climent, N., Conocimiento sobre orientación espacial en estudiantes de E.S.O., in Investigación en Educación Matemática XIV by M. Moreno, A. Estrada, J. Carrillo y T.A. Sierra, 581-596, SEIEM, Lleida, España (2010)

Young, C.J., Levine, S.C., y Mix, K.S., The connection between spatial and mathematical ability across development, https://doi.org/10.3389/fpsyg.2018.00755, Frontiers in Psychology, 9, 755 (2018) 